\title{
The turbulent spectrum created by non-Abelian plasma instabilities
}

\author{
Peter Arnold \\ Department of Physics, University of Virginia, \\ Box 400714, Charlottesville, Virginia 22901, USA \\ Guy D. Moore \\ Department of Physics, McGill University, \\ 3600 University St., Montréal QC H3A 2T8, Canada
}

(Dated: September 21, 2005)

\begin{abstract}
Recent numerical work on the fate of plasma instabilities in weakly-coupled non-Abelian gauge theory has shown the development of a cascade of energy from long to short wavelengths. This cascade has a steady-state spectrum, analogous to the Kolmogorov spectrum for turbulence in hydrodynamics or for energy cascades in other systems. In this paper, we theoretically analyze processes responsible for this cascade and find a steady-state spectrum $f_{\boldsymbol{k}} \sim k^{-2}$, where $f_{\boldsymbol{k}}$ is the phase-space density of particles with momentum $\boldsymbol{k}$. The exponent -2 is consistent with results from numerical simulations. We also discuss implications of the emerging picture of instability development on the "bottom-up" thermalization scenario for (extremely high energy) heavy ion collisions, emphasizing fundamental questions that remain to be answered.
\end{abstract}




\section{INTRODUCTION}

It is important to understand theoretically the mechanisms by which quark-gluon plasmas can locally equilibrate in heavy ion collisions such as those at RHIC. Since the path-breaking work of Baier, Mueller, Schiff and Son [1] on "bottom-up" thermalization, one modest theoretical goal has been to understand the process of equilibration in the simplifying theoretical limit of arbitrarily high-energy collisions, where the running strong coupling $\alpha_{\mathrm{s}}$ can be treated as small. Typically, theorists expect to be able to solve weakly-coupled problems, but equilibration of weakly-coupled non-Abelian plasmas has proven to be a very rich and challenging problem. In particular, the original attempt by Baier et al. did not account for the physics of plasma instabilities, which are now believed to play a crucial role during some of the early stages of thermalization [2, 3, 3 , .

Early in a heavy-ion collision, particles have an anisotropic distribution of momenta, as measured in local frames moving with the expanding plasma [1]. Later, they scatter and equilibrate to locally isotropic distributions, giving rise to hydrodynamic behavior. Generically, anisotropic distributions of particles produce collective plasma instabilities known as Weibel or filamentary instabilities [5, 6, 7, 8, 9, 10, 11]. These instabilities are associated with filamentation of particle currents and initially exponential growth of long-wavelength magnetic fields. Since these magnetic fields in turn deflect the particles in the plasma, and so perhaps drive the particles towards isotropization and equilibration, it has been important to understand just how large these unstable magnetic fields grow [12, 13, 14, 15, 16, 17].

In this paper, we will follow the nomenclature of Baier et al. and refer to the initial particles of the plasma (e.g. small $x$ gluons in the saturation picture) as "hard particles," and we will refer to their momentum scale (e.g. the saturation scale) as $p_{\text {hard }}$. We will refer to the momentum scale of magnetic plasma instabilities as $m_{\text {soft }}$. Once the system has expanded enough that the hard partons have perturbative density $\left(n_{\text {hard }} \ll p_{\text {hard }}^{3} / g^{2}\right)$, one generically finds that $m_{\text {soft }} \ll p_{\text {hard }}[2,13]$. We will assume and exploit this hierarchy of scales in what follows. We will refer to any scale that is parametrically small compared to $p_{\text {hard }}$ as "soft."

Fig. 11 shows a cartoon of the results of numerical studies in $3+1$ dimensions of nonAbelian plasma instability growth [16, 17] in a non-expanding system. The energy in soft magnetic fields is plotted versus time for simulations initiated with a very tiny magnetic seed field. The soft magnetic field energy initially grows exponentially, but the behavior changes once the fields become large enough that their non-Abelian self-interactions become non-perturbative $\left(B^{2} \sim m_{\text {soft }}^{4} / g^{2}\right)$. The energy growth then becomes linear with time. For a non-expanding system, this linear growth would continue until some parametrically long time later (not addressed by the simulations) when the soft fields eventually have a nonperturbatively large effect on the original, anisotropic hard particles that created them.

Recent numerical work [18] indicates that the linear growth in energy is associated with a cascade of energy from the soft, perturbatively unstable modes toward the ultraviolet. A cartoon of the development of the spectrum is shown in Fig. 2. (a) The system starts with the initial, anisotropic hard particles of momentum $\sim p_{\text {hard }}$ plus some small initial fluctuations in softer modes. (b) Plasma instabilities initially lead to rapid, exponential growth of unstable soft modes of momenta $\sim m_{\text {soft }}$. (c) The unstable modes stop growing in amplitude once they become non-perturbatively large, but total soft energy continues to grow (linearly) as interactions feed power from $m_{\text {soft }}$ into increasingly higher momentum modes. The spectrum of this cascade falls with some characteristic power $k^{-\nu}$, and the 


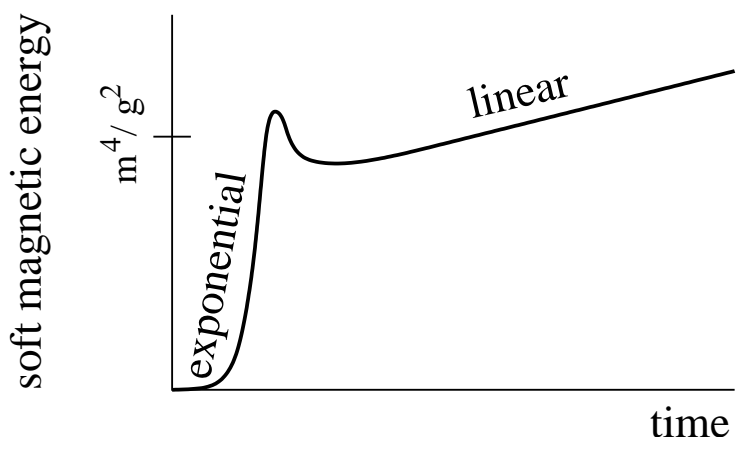

FIG. 1: A qualitative depiction of soft magnetic field energy vs. time for the growth of non-Abelian magnetic instabilities.

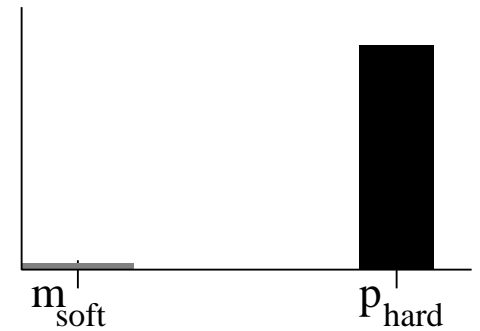

(a)

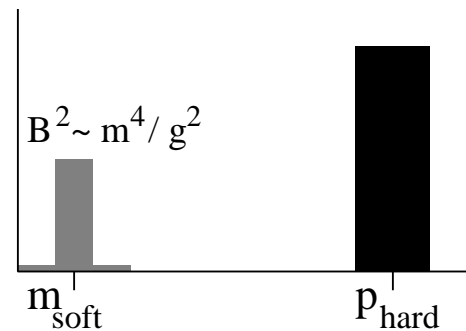

(b)

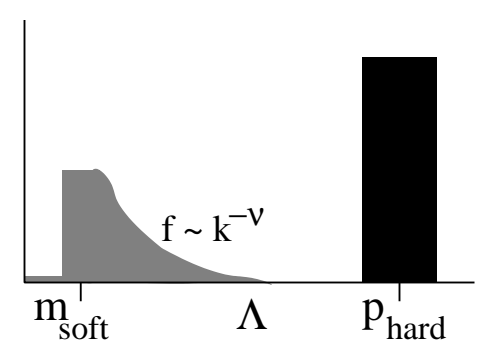

(c)

FIG. 2: A cartoon description of the energy spectrum of excitations at various times during the development of non-Abelian plasma instabilities.

upper momentum limit $\Lambda(t)$ of the cascade increases with time. The goal of the present paper is to theoretically determine the exponent $\nu$ by considering the processes responsible for this cascade.

The basic plan of our analysis will be somewhat similar to investigations of thermalization during reheating after inflation in the early Universe [19, 20, 21]. The details of our problem, and the result for the spectral index $\nu$, are different, however.

Let $f_{\boldsymbol{k}}$ be the average occupancy of each mode $\boldsymbol{k}$. In analyzing the cascade, we shall take

$$
1 \ll f_{k} \ll \frac{1}{g^{2}}
$$

for $m_{\text {soft }} \ll k \ll \Lambda(t)$. In this range of $f$, the physics is perturbative $\left(f_{\boldsymbol{k}} \ll 1 / g^{2}\right)$ and can be equally well described by a kinetic theory of particles or by a classical theory of fields $\left(f_{\boldsymbol{k}} \gg 1\right)[22] .{ }^{1}$ The perturbative nature of the cascade for $k \gg m_{\text {soft }}$ follows from (i) the non-perturbative occupation number $f \sim 1 / g^{2}$ at the instability scale $m_{\text {soft }}$ and (ii) the

\footnotetext{
${ }^{1}$ This is a slight oversimplification. Perturbative occupation numbers $f_{\boldsymbol{k}} \ll 1 / g^{2}$ are necessary but not sufficient for a kinetic theory description of relativistic systems. For quantitatively accurate (or qualitative order-of-magnitude) applications of kinetic theory, one must check that deBroglie wavelengths and the duration of scattering events are $\ll($ or $\lesssim)$ mean free paths, for whatever processes dominate the kinetic description.
} 
decrease of $f$ as some power $k^{-\nu}$ to be determined. This gives

$$
f_{\boldsymbol{k}}^{\mathrm{ss}} \sim \frac{1}{g^{2}}\left(\frac{m_{\mathrm{soft}}}{k}\right)^{\nu}
$$

where the superscript "ss" stands for "steady-state." Perturbative cascades are known in the plasma physics literature as "weak plasma turbulence" [23]. We will restrict attention to the case where $\Lambda \ll g^{-2 / \nu} m_{\text {soft }}$ in order that (1.2) satisfy the other condition $f_{\boldsymbol{k}} \gg 1$. This classical field theory limit is implicit to the numerical simulations which discovered the cascade [16, 17, 18], since these simulations treated all soft fields as classical.

In the next section, we warm up by discussing $2 \rightarrow 2$ scattering of particles at the UV end of the cascade. We show that this process alone would reproduce a result for the spectral index $\nu$ in (1.2) known from analogous scalar theory applications $(\nu=5 / 3)$. In Sec. III, we show that there is a more important process: multiple scattering from the non-perturbative infrared background produces a steeper distribution, $\nu=2$. This result is consistent with numerical simulations [18]. Sec. IV] discusses the back reaction the cascade has on the infrared physics of the instability and poses some open questions concerning logarithmic effects in our analysis. In Sec. V] we return to the question of how instabilities affect the bottom-up scenario and discuss what remains to be understood about instability development before this question can be answered. Finally, we offer our conclusions.

Non-perturbative numerical studies of instability development in $3+1$ dimensions have only studied hard particle distributions which are moderately, but not extremely, anisotropic [16, 17, 18. In the case of moderate, $O(1)$ anisotropy, there is a single scale which characterizes $m_{\mathrm{soft}}$, given parametrically by [2, 10, 11]

$$
m_{\text {soft }}^{2} \sim g^{2} \int_{\boldsymbol{p}} \frac{f_{\boldsymbol{p}}^{\text {hard }}}{p},
$$

where the integral is over the distribution of hard particle momenta $\boldsymbol{p}$. For extremely anisotropic distributions, the situation is more complicated. In most of this paper, we will implicitly assume that there is only a single relevant soft scale. We will only discuss the unresolved issues surrounding extremely anisotropic distributions in our discussion of bottom-up thermalization in Sec. D.

\section{WARM-UP: HARD $2 \rightarrow 2$ SCATTERING}

The standard picture of driven turbulence is that something pumps energy into the cascade from the infrared (IR) end at a constant rate. In the absence of dissipation, this leads to linear growth in the total energy. At the same time, energy is flowing out the ultraviolet (UV) end by increasing $\Lambda$. The spectral index $\nu$ of the cascade can be determined by balancing (i) the rate at which energy flows out the UV end of the cascade by scattering processes that extend the UV cascade by increasing $\Lambda$ (e.g. scattering momentum $\Lambda$ particles to produce momentum $2 \Lambda$ particles) and (ii) the constant rate energy enters the IR end of the cascade.

As a review and warm-up example, let us for the moment (incorrectly) assume that the dominant process for increasing the cut-off was large-angle $2 \rightarrow 2$ scattering with all momenta roughly of order $\Lambda$. Fig. 3 shows an example of how two particles with momenta $p_{1}$ and $p_{2}$ less than $\Lambda$ can produce a particle with momentum greater than $\Lambda$ in $2 \rightarrow 2$ scattering. Now 
consider what the time scale would be for bringing $f_{2 \Lambda}$ up to its steady-state value (1.2). For a statistically homogeneous system, the Boltzmann equation for $2 \rightarrow 2$ scattering has the form

$$
\frac{d}{d t} f\left(p_{4}\right)=\int_{\boldsymbol{p}_{1} \boldsymbol{p}_{2} \boldsymbol{p}_{3}}\left|\mathcal{M}_{\boldsymbol{p}_{1} \boldsymbol{p}_{2} \leftrightarrow \boldsymbol{p}_{3} \boldsymbol{p}_{4}}\right|^{2}\left[f_{1} f_{2}\left(1+f_{3}\right)\left(1+f_{4}\right)-f_{3} f_{4}\left(1+f_{1}\right)\left(1+f_{2}\right)\right]
$$

for bosons such as the soft gluons that make up our cascade. In the $f \gg 1$ classical field limit of (1.1), this becomes

$$
\frac{d}{d t} f\left(p_{4}\right)=\int_{\boldsymbol{p}_{1} \boldsymbol{p}_{2} \boldsymbol{p}_{3}}\left|\mathcal{M}_{\boldsymbol{p}_{1} \boldsymbol{p}_{2} \leftrightarrow \boldsymbol{p}_{3} \boldsymbol{p}_{4}}\right|^{2}\left[f_{1} f_{2}\left(f_{3}+f_{4}\right)-f_{3} f_{4}\left(f_{1}+f_{2}\right)\right] .
$$

Even if $f\left(p_{4}\right)$ were initially small for $p_{4}>\Lambda$ as in Fig. 3. there is a term in this equation with $f_{1} f_{2} f_{3} \sim f_{\Lambda}^{3}$. Take the square-amplitude $|\mathcal{M}|^{2}$ for large-angle scattering as order $g^{4}$ times normalizations and the energy-momentum conserving delta function. The characteristic rate $\Gamma$ implied by (2.2) for $f(2 \Lambda)$ to grow to its steady state value $f_{2 \Lambda}^{\text {ss }} \sim f_{\Lambda}^{\text {ss }}$ is then given by

$$
\Gamma f_{\Lambda}^{\mathrm{ss}} \sim g^{4}\left(f_{\Lambda}^{\mathrm{ss}}\right)^{3} \Lambda
$$

where the factor of $\Lambda$ on the right-hand side follows from dimensional analysis. So

$$
\Gamma \sim g^{4}\left(f_{\Lambda}^{\mathrm{ss}}\right)^{2} \Lambda
$$

From our assumed power-law form (1.2) for $f^{\mathrm{ss}}$, this gives

$$
\Gamma \propto \Lambda^{1-2 \nu} .
$$

Since the cutoff $\Lambda$ has to change on the time scale of the age $t$ of the system, we must have $\Gamma \sim 1 / t$.

On the other hand, let us estimate the rate from the linear growth of the total energy density in the cascade. Using (1.2), the total energy density is

$$
\epsilon \sim \int d^{3} k k f_{\boldsymbol{k}} \sim \frac{\Lambda^{4}}{g^{2}}\left(\frac{m_{\mathrm{soft}}}{\Lambda}\right)^{\nu} \propto \Lambda^{4-\nu}
$$

(provided $\nu<4$, which we will find). Taking $\epsilon \propto t$, the corresponding rate is

$$
\frac{1}{t} \propto \Lambda^{\nu-4}
$$

Comparing the exponent here to (2.5), we would obtain $\nu=5 / 3$, in agreement with previous analysis in the literature of certain cascades in field theory where large-angle $2 \rightarrow 2$ scattering is the dominant process [21]. ${ }^{2}$

\footnotetext{
${ }^{2}$ In particular, this $\nu$ agrees with Eq. (66) of Ref. [21].
} 


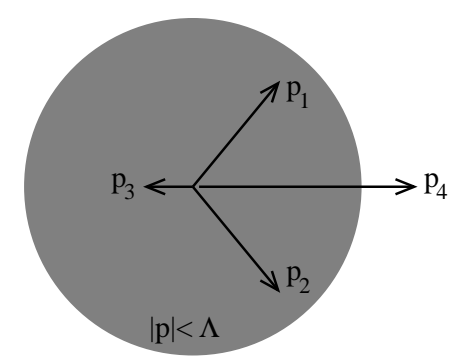

FIG. 3: Momenta in a $\boldsymbol{p}_{1} \boldsymbol{p}_{2} \rightarrow \boldsymbol{p}_{3} \boldsymbol{p}_{4}$ process that creates a particle with momentum larger than $\Lambda$.

\section{THE DOMINANT PROCESS}

The more efficiently the system can scatter energy into the UV, the larger will be the exponent $\nu$. The goal is to identify the most efficient process. We propose that the dominant process is multiple scattering of quanta of momentum $\Lambda$ off of the non-perturbative background field of wave-number $m_{\text {soft }}$. In this section, we analyze this process and show that it is more important than the large-angle $2 \rightarrow 2$ scattering considered in the last section.

Consider a relatively high-energy particle $\left(k \sim \Lambda \gg m_{\text {soft }}\right)$ propagating through a soft, random, non-perturbatively large background field $A_{\text {bkgd }}$ having wave-numbers of order $m_{\text {soft }}$. The particle can scatter from the background as in Fig. 4. Non-perturbatively large means $g A_{\text {bkgd }} \sim m_{\text {soft }}$, and so the only parameters in the problem are $k$ and $m_{\text {soft }}$. The probability of interaction is independent of $k$ in the $k \gg m_{\text {soft }}$ (Eikonal) limit. By dimensional analysis, the rate is therefore $O\left(m_{\text {soft }}\right)$ to change momentum by $O\left(m_{\text {soft }}\right)$. Each such scattering will have a perturbative effect on the particle, randomly changing its momentum $k$ by $m_{\text {soft }} \ll k$. $N$ successive collisions will change $k$ by order $N^{1 / 2} m_{\text {soft }}$ in a time of order $N / m_{\text {soft }}$. Taking $N \sim\left(k / m_{\text {soft }}\right)^{2} \sim\left(\Lambda / m_{\text {soft }}\right)^{2}$, we see that the time taken to change particle momenta from $\Lambda$ to $2 \Lambda$ at the UV end of the cascade would be

$$
t \sim\left(\frac{\Lambda}{m_{\mathrm{soft}}}\right)^{2} \frac{1}{m_{\mathrm{soft}}},
$$

corresponding to a net rate that depends on $\Lambda$ as

$$
\Gamma_{\Lambda \rightarrow 2 \Lambda} \sim \frac{1}{t} \propto \Lambda^{-2}
$$

If we equate this to the rate (2.7) determined by linear energy growth, we obtain the spectral index

$$
\nu=2 \text {. }
$$

The fact that this $\nu$ is larger than the $\nu=5 / 3$ of Sec. II indicates that the large-angle $2 \rightarrow 2$ processes of Fig. 3 are unimportant compared to multiple scattering by Fig. $4{ }^{3}$

We note in passing that interactions with a non-perturbative background field have also been important in the study of cascades during reheating of scalar field theories in the

\footnotetext{
${ }^{3}$ One can check this explicitly by comparing the rates for both $\nu=5 / 3$ and $\nu=2$ : Eq. (2.4) gives $m_{\text {soft }}\left(m_{\text {soft }} / \Lambda\right)^{2 \nu-1}$ for large-angle $2 \rightarrow 2$, and Eq. (3.1) gives in both cases a parametrically larger rate $m_{\text {soft }}\left(m_{\text {soft }} / \Lambda\right)^{2}$ for multiple scattering off of the non-perturbative background.
} 


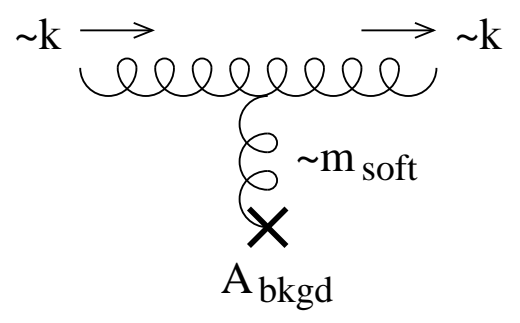

FIG. 4: A particle with momentum $k \gg m_{\text {soft }}$ scattering off of the non-perturbative field modes with wavenumber $m_{\text {soft }}$.

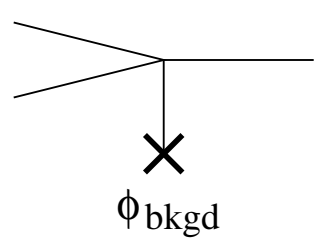

(a)

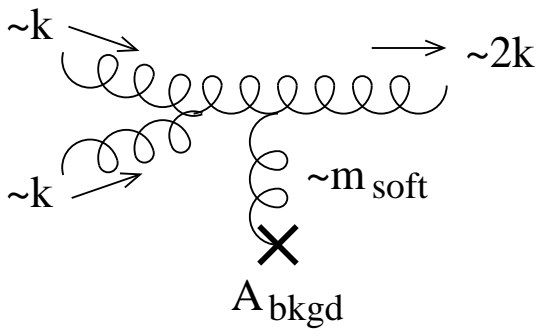

(b)

FIG. 5 : $2 \rightarrow 1$ processes catalyzed by a background field in (a) scalar $\phi^{4}$ theory and (b) gauge theory (inverse bremsstrahlung).

background of an oscillating inflation field [19, 21]. However, processes analogous to Fig. 4 were not relevant in that case because the background field carried zero momentum and so could not directly transfer momentum to the particles. In that case, the dominant process was Fig. 5a, which is not as efficient, giving a smaller value of $\nu$ in those applications. For our application, we consider in the Appendix the related process of Fig. 5b: $2 \rightarrow 1$ inverse bremsstrahlung of particles with momenta $k \gg m_{\text {soft }}$, catalyzed by the non-perturbative background field. We find that, due to the Landau-Pomeranchuk-Migdal (LPM) effect, this process is not competitive with multiple scattering via Fig. 4.

Failing to find a more efficient process than multiple scattering off of the non-perturbative background, our provisional conclusion is that the spectral index $\nu$ should be 2 . This is consistent with results from simulations [18], which extract $\nu=2 \pm 0.2$ from Coulomb-gauge spectra of the cascade. From (2.7), we also get that the UV end of the cascade should grow as

$$
\Lambda(t) \propto t^{1 /(4-\nu)} \sim t^{1 / 2}
$$

Using (1.2), this means that our classical treatment $\left(f_{k} \gg 1\right.$ for $\left.k \ll \Lambda\right)$ is valid for times $t \lesssim 1 / g^{2} m_{\text {soft }}$.

\section{SCREENING, SCATTERING, AND INTERNAL CONSISTENCY}

Having proposed a cascade with

$$
f^{\mathrm{ss}}(k) \sim \frac{1}{g^{2}}\left(\frac{m_{\mathrm{soft}}}{k}\right)^{2}
$$


we should now ask whether the development of the cascade might have a back-reaction on the dynamics of the infra-red (IR) modes with wave-numbers of order $m_{\text {soft }}$. These IR modes are the starting point for energy to enter the cascade, and one could imagine that a backreaction might conceivably change the rate at which energy is taken into the cascade from the original anisotropic hard particles. If such a change to the rate depended on $\Lambda(t)$ and so on time, it would be harder to understand why the energy in the cascade grows linearly with time, as indicated by numerical simulations.

Let us investigate how the cascade affects the soft scale $m_{\text {soft }}$ itself, given by (1.3). This scale is initially created by the hard particle distribution, as we have indicated explicitly in (1.3). However, once the cascade develops, its excitations could also contribute:

$$
\Delta m^{2} \sim g^{2} \int_{\boldsymbol{k}} \frac{f_{\boldsymbol{k}}^{\text {cascade }}}{k} \sim g^{2} \int_{\sim m_{\mathrm{soft}}}^{\sim \Lambda(t)} d k k f_{k}
$$

where $\boldsymbol{k}$ is integrated over momenta in the cascade. How big is this contribution, and is its dependence on time through $\Lambda(t)$ significant? For $\nu<2$, the integral (4.2) would be sensitive to large $\Lambda(t)$; for $\nu>2$, it would not. The spectral index $\nu=2$ found in this paper is the borderline case, and (4.1) produces

$$
\Delta m^{2} \sim m_{\mathrm{soft}}^{2} \ln \left(\frac{\Lambda(t)}{m_{\mathrm{soft}}}\right) .
$$

If we ignore the logarithm, then $\Delta m^{2} \sim m_{\text {soft }}^{2}$, and the picture can be self-consistent. The creation of the cascade would make an $O(1)$ relative change to the IR physics driving the instability, and so could make an $O(1)$ relative change to the rate of linear energy growth. That still allows the system to settle down into steady growth as $t \rightarrow \infty$, consistent with numerical results.

The formal logarithmic dependence of (4.3) on $\Lambda(t)$, however, suggests that the energy growth rate should see logarithmic in time corrections. Current simulations [18] do not support such logarithmic corrections, perhaps because the coefficient happens to be small, and perhaps because of the limited statistical power of existing simulations. In addition, the above analysis was rather crude: the details of exactly how $\Delta m^{2}$ affects instability development depend, for instance, on the degree of anisotropy of the cascade particles as a function of time. ${ }^{4}$ Thoroughly understanding the physics of the cascade at the level of logarithms remains an open problem.

\footnotetext{
${ }^{4}$ For instance, isotropic distributions do not produce magnetic instabilities. Adding a large isotropic contribution $f^{\text {cascade }}$ to an anisotropic hard particle distribution $f^{\text {hard }}$ will not change the momentum scale of the instability. It will, however, decrease the perturbative growth rate of such instabilities. (See, for example, the discussion in Ref. [4].) Roughly speaking, this is because changing magnetic fields require electric fields, the ability of a medium to support electric fields depends on its conductivity, and the conductivity is affected by the number of isotropic as well as anisotropic particles. On the other hand, if $f$ cascade is significantly anisotropic, it could increase the momentum scale of the instability, which would tend to increase growth rates. Numerical simulations suggest that the excitations in the cascade are somewhat but not perfectly isotropic. A simulation in Ref. 18], for a particular hard particle distribution, found that the magnetic fields making up the excitations in the cascade had $B_{z}^{2}$ equal to roughly $80 \%$ of $B_{x}^{2}$ and $B_{y}^{2}$.
} 
We see that cascade particles can have an $O(1)$ effect on the infrared dynamics (up to logarithms). In previous work [24], we argued that particles can make two important contributions to the behavior of the plasma. One is the effect on infrared fields ("screening," in the parlance of Ref. [24]). The other is the contribution to the scattering rate for hard particles. That is, when a hard particle (or another cascade particle) scatters, what fraction of the time is it scattering from a cascade particle? This is determined by the relative contributions to the integral

$$
\int_{k} f_{\boldsymbol{k}}\left(1+f_{\boldsymbol{k}}\right)
$$

where $\left(1+f_{\boldsymbol{k}}\right)$ is a final state Bose stimulation factor and where we will focus on $k$ 's in the cascade. For our $f \propto k^{-2}$ cascade (with $f \gg 1$ ), the integral is of form

$$
\int_{\sim m_{\mathrm{soft}}}^{\sim \Lambda} k^{-4} k^{2} d k,
$$

which is dominated by the smallest momenta and insensitive to the moving cutoff $\Lambda(t)$. That is, the cascade particles are not important as scatterers. Most scattering is off the $m_{\text {soft }}$ nonperturbative background, not off the higher-momentum fluctuations. This is another way of seeing why it is scatterings from the soft background, rather than between cascade particles, which determine the progress of the cascade.

\section{WHITHER BOTTOM-UP THERMALIZATION?}

We introduced this paper with a discussion of how nonabelian plasma instabilities play a significant role in the bottom-up scenario for quark-gluon plasma equilibration in the high energy limit. We now have a reasonable picture of the development of non-Abelian plasma instabilities in a non-expanding system. Once we understand, at least parametrically, the basic processes and their rates, we should be able to apply this knowledge to an analysis of expanding plasmas. Unfortunately, there is an obstacle. For reasons of computational practicality, simulations have been performed for moderately, but not extremely, anisotropic hard particle distributions. For moderate anisotropy, there is a single parametric scale $m_{\text {soft }}$ for IR physics. Non-perturbative IR magnetic fields are therefore $O\left(m_{\text {soft }}^{2} / g^{2}\right)$, the linear growth of soft energy density is $O\left(m_{\text {soft }}^{5} t / g^{2}\right)$, and so forth. However, as we shall review, the first stages of the bottom-up scenario involve extremely anisotropic hard particle distributions, where (in local fluid frames) the angle $\Delta \theta$ between typical particle velocities and the transverse plane is parametrically small. One must therefore understand the parametric dependence of instability development on this additional small parameter, $\Delta \theta$. We leave such understanding for future work. In this section, we simply wish to emphasize the need by showing how the current state of uncertainty can have a significant impact on the first stage of bottom-up thermalization. In particular, we will consider a possible alternative to the recent analysis of the effects of instabilities by Bödeker [4].

In their original analysis of the stages of thermalization for arbitrarily high energy heavyion collisions, Baier et al. [1] considered an initial, non-perturbatively dense state of saturated low- $x$ gluons produced in the collision. In their analysis, these were labeled the "hard" partons in the plasma, with momenta $p_{\text {hard }}$ of order the saturation scale $Q_{\mathrm{s}}$. Their analysis is restricted to following the process of thermalization for $\tau \gg 1 / Q_{\mathrm{s}}$, where $\tau$ is proper time since the collision. 
They assume initial conditions at time $\tau \sim 1 / Q_{\mathrm{s}}$ with number density $n \sim Q_{\mathrm{s}}^{3} / g^{2}$ of such hard particles. This number density dilutes as $n \sim\left(Q_{\mathrm{s}} \tau\right)^{-1} Q_{\mathrm{s}}^{3} / g^{2}$ due to the one dimensional expansion of the system. The scale (1.3) characterizing infrared physics (which they interpreted as a Debye screening scale) then evolves with time as

$$
m_{\infty}^{2} \sim g^{2} \int_{p} \frac{f_{p}}{p} \sim \frac{g^{2} n}{Q_{\mathrm{s}}} \sim Q_{\mathrm{s}}^{2}\left(Q_{\mathrm{s}} \tau\right)^{-1} .
$$

Our notation " $m_{\infty}$ " for this scale is in preparation for our later discussion of plasma instabilities, for notational consistency with previous work. ${ }^{5}$

In the first phase of their analysis, the gluons dilute to perturbative densities as the system expands, and their local distributions of velocity temporarily become highly anisotropic due to momentum selection: over time, approximately free-streaming particles with the same $v_{z}$ would move to approximately the same displacement $z$, along the beam axis, from the origin of the collision. This drive towards local anisotropy is moderated only by processes which scatter the hard particles and so try to isotropize them. Baier et al. considered random, individual, small-angle $2 \rightarrow 2$ scattering of the hard particles and found that, early in their scenario, the balance between expansion and scattering effects produces local distributions with anisotropy

$$
\frac{p_{z}}{p_{\perp}} \sim \frac{1}{\left(Q_{\mathrm{s}} \tau\right)^{1 / 3}}
$$

in local fluid rest frames. This result indicates extreme anisotropy for $Q_{\mathrm{s}} \tau \gg 1$. Later, however, once the system dilutes enough that $f_{\text {hard }} \lesssim 1$, Baier et al. found that other processes come into play which eventually locally isotropize and thermalize the system. Here, we modestly focus our attention on the first phase, described by (5.2) in the original scenario. ${ }^{6}$

Arnold, Lenaghan, and Moore [2] pointed out that the first phase will be modified by plasma instabilities. Recently, Bödeker [4] has attempted to deduce how scattering of hard particles from soft fields created by plasma instabilities will modify (15.2). Bödeker assumed that soft fields consist only of a non-perturbative component $\left(f_{\text {soft }} \sim 1 / g^{2}\right)$ with momenta $m_{\text {soft }} \sim m_{\infty}$ given by (1.3). For hard particle distributions with $O(1)$ anisotropy, this is parametrically the only characteristic scale of unstable modes. With these assumptions, Bödeker found

$$
\frac{p_{z}}{p_{\perp}} \sim \frac{\left(m_{\mathrm{soft}}^{3} \tau\right)^{1 / 2}}{Q_{\mathrm{s}}} \sim \frac{1}{\left(Q_{\mathrm{s}} \tau\right)^{1 / 4}},
$$

which is parametrically larger than (5.2). This estimate is in keeping with the discussion of this paper; the dominant way in which high momentum particles (whether in the cascade, as we have discussed, or even higher momentum, as is relevant here) undergo scattering, is by interaction with the nonperturbative fields at the scale $m_{\text {soft }}$.

However, this estimate is not secure, because the treatment of the unstable scale $m_{\text {soft }}$ has been made assuming $O(1)$ anisotropy, yet it predicts a parametrically large anisotropy for times $\tau \gg 1 / Q_{\mathrm{s}}$. Therefore we must reconsider the behavior of the instability for highly anisotropic systems, a point also emphasized by Bödeker [4]. As we shall review, there

\footnotetext{
5 The historical reason for the subscript " $\infty$ " is that, in addition to its role in the physics of instabilities, $m_{\infty}$ turns out to be the mass scale of transverse plasmons in the large momentum limit $($ i.e. $p \rightarrow \infty)$ 24].

6 This corresponds to $1 \ll Q_{\mathrm{s}} \tau \ll \alpha_{\mathrm{s}}^{-3 / 2}$ in the original scenario of Ref. 1].
} 
is another characteristic scale $q_{\max } \gg m_{\infty}$ for such extremely anisotropic distributions. We believe that Bödeker's estimate of taking $m_{\text {soft }}$ of order the lower scale $\left(m_{\infty}\right)$ in (5.3) probably provides a lower bound on the result for $p_{z} / p$. We will not attempt to make a complete treatment here, but will provide what we believe is a sensible parametric upper bound.

For highly anisotropic, oblate distributions of hard particles, the spread of angles of hard particle momenta $\boldsymbol{p}$ about the transverse plane is characterized by

$$
\Delta \theta \sim \frac{p_{z}}{p} .
$$

The perturbatively unstable modes are described by two scales. One is $m_{\infty}$, defined before, whose inverse represents the time scale that a gauge field must act on the particles before the back-reaction of particles on the field becomes important. $m_{\infty}$ characterizes the growth rate of instabilities. The other scale is the largest wave vector $q$ of any unstable soft gauge field mode, which is

$$
q_{\max } \sim \frac{m_{\infty}}{\Delta \theta} .
$$

For a detailed explanation of how these scales arise, see Ref. [2]; here we give a physical argument. For a gauge field mode to be unstable, the hard particles must fly for a time scale $\sim m_{\infty}^{-1}$ in the presence of that mode, and be deflected in the same direction that whole time. That means that the magnetic field must be roughly the same all along the trajectory of the particle's path. Therefore, the gauge field must take roughly the same value over distances $\delta x_{\perp} \sim 1 / m_{\infty}$ in the two transverse directions, and over a distance $\delta z \sim \Delta \theta / m_{\infty}$ in the longitudinal direction (since this is how far a typical particle moves in the longitudinal direction in that time scale). That means that modes with $q_{\perp} \lesssim m_{\infty}$ and $q_{z} \lesssim m_{\infty} / \Delta \theta$ are unstable. This agrees with a more detailed analysis 2].

How large can the soft fields grow? We can place an upper bound as follows. Gauge fields in different color directions are all growing simultaneously. When these grow large enough that they can randomize the color of a hard excitation in a time scale $\sim 1 / m_{\infty}$, then the hard modes will no longer be contributing to the time development of the unstable modes on the time scale required to make the instability work. The rotation of color charge as a hard particle moves along a trajectory is a matrix given by the Wilson line

$$
\mathcal{P} \exp \left[i g \int d x \cdot A\right]
$$

where $\mathcal{P}$ represents path ordering. In the analysis of a particular unstable mode, think of all the other unstable modes as background. The color rotation (5.6) due to a noncommuting background field will therefore be significant, and so stop instability growth, if $g \delta x \cdot A \gtrsim 1$. To avoid color randomization of generic hard particles in our extremely anisotropic distribution, we therefore need, for example, ${ }^{7,8}$

$$
A_{\perp} \lesssim \frac{1}{g \delta x_{\perp}} \sim \frac{m_{\infty}}{g} .
$$

\footnotetext{
${ }^{7}$ In order to discuss parametric estimates concerning the gauge field $A$, one should assume that one is in a gauge where $A$ is relatively smooth. Here, we imagine that we study the state of the system at a particular time $t$ by (i) working in $A_{0}=0$ gauge, and then (ii) making a spatial gauge transformation to make $\boldsymbol{A}$ as smooth as possible at that particular time.

${ }^{8}$ Another way to arrive at the relation (5.7) is to consider the propagator $\left(D_{0}+\boldsymbol{v} \cdot \boldsymbol{D}\right)^{-1}$ of particle
} 
Since the dominant instabilities have $\boldsymbol{q}$ nearly in the $z$ direction, with $|\boldsymbol{q}| \sim q_{\max }$, this implies that an upper bound on how large the corresponding magnetic field strength can grow is ${ }^{9}$

$$
B \sim q_{\max } A_{\perp} \sim \frac{q_{\max } m_{\infty}}{g} \sim \frac{m_{\infty}^{2}}{g \Delta \theta},
$$

coherent on transverse length scales of $m_{\infty}^{-1}$. Such fields will give momentum kicks to the hard modes due to the $g \boldsymbol{v} \times \boldsymbol{B}$ term in the Lorentz force law. The force is coherent over the time scale $\delta t \sim m_{\infty}^{-1}$ (since this is both how often $B$ changes as one moves in the transverse direction, and how often the particle's charge gets rotated). The individual momentum kicks are of size $\Delta p_{z} \sim g B \delta t \sim q_{\max }$, and they lead to momentum diffusion in the $p_{z}$ direction of size

$$
p_{z}^{2} \sim \frac{\tau}{\delta t}\left(\Delta p_{z}\right)^{2} \sim q_{\max }^{2} m_{\infty} \tau
$$

Therefore,

$$
\frac{p_{z}}{p_{\perp}} \sim \frac{\left(q_{\max }^{2} m_{\infty} \tau\right)^{1 / 2}}{Q_{\mathrm{s}}} \sim \frac{\left(m_{\infty}^{3} \tau\right)^{1 / 2}}{Q_{\mathrm{s}} \Delta \theta} .
$$

[Compare to (5.3).] Substituting into the defining relation (5.4) for $\Delta \theta$, and using the relation (5.1) for the time development of $m_{\infty}$, gives

$$
\frac{p_{z}}{p_{\perp}} \sim \sqrt{\frac{\left(m_{\infty}^{3} \tau\right)^{1 / 2}}{Q_{\mathrm{s}}}} \sim \frac{1}{\left(Q_{\mathrm{s}} \tau\right)^{1 / 8}} .
$$

The above estimate is correct if our picture of how plasma instabilities are cut off in a highly anisotropic setting proves correct. While we believe that our estimates represent an upper bound on such a cutoff, we are not confident that they are correct. The moral of this story is that better theoretical understanding is needed of the non-perturbative dynamics of instabilities in the case of extremely anisotropic distributions. Until then, it will be difficult to analyze even the first phase of bottom-up thermalization with complete certainty.

\section{CONCLUSIONS}

We have argued that, in the presence of non-Abelian instabilities that have grown nonperturbatively large, the spectrum of the cascade of soft field energy from unstable modes into the ultra-violet should have the form $f_{\boldsymbol{k}} \sim k^{-\nu}$ with $\nu=2$. This is consistent with results from numerical simulations.

We should emphasize that this turbulent cascade is only a transitory phenomenon appearing during the local thermalization of the quark-gluon plasma in the high energy limit. It is not directly related to the spectrum of particles that much later leave the collision after

fluctuations in non-Abelian Vlasov equations. In perturbative formulas for instabilities (e.g. Refs. 2, 10, 11]), such propagators appear in the perturbative form $\left(\partial_{0}+\boldsymbol{v} \cdot \boldsymbol{\nabla}\right)^{-1} \sim(\omega-\boldsymbol{v} \cdot \boldsymbol{q})^{-1}$. One can then ask when the $g \boldsymbol{v} \cdot \boldsymbol{A}$ in $\boldsymbol{v} \cdot \boldsymbol{D}$ can be treated as a perturbation to $\omega$ and $\boldsymbol{v} \cdot \boldsymbol{q} \sim q_{\perp}+\Delta \theta q_{z}$, both of which are order $m_{\infty}$.

${ }^{9} q_{\perp} \sim m_{\infty}$. Similar reasoning to (5.7) could allow $A_{z} \sim 1 /(g \delta z) \sim q_{\max } / g$, which gives an $O\left(q_{\perp} A_{z}\right)$ contribution to $B$ that is the same size as (5.8). 
hadronization. Also, though the word "turbulence" is used, the existence of this cascade does not mean that the plasma is described by ideal hydrodynamics at the time the cascade is formed. At the early times considered in this paper, most of the energy that will become the quark-gluon plasma is still in hard particles, $p_{\text {hard }} \sim Q_{\mathrm{s}}$, which have not yet thermalized and which are locally extremely anisotropic.

It would be delightful to be able to apply the current understanding of plasma instability development to produce a complete picture of thermalization in the high energy limit, finally completing the program initiated by the original bottom-up scenario. A lot of progress has been made over the last year in understanding the qualitative behavior and parametric dependence of the non-perturbative physics of instabilities for the case of moderately anisotropic hard particles. Unfortunately, figuring out the detailed role of instabilities in bottom-up thermalization will likely require at least a similar level of understanding of the case of extremely anisotropic hard particles, which have not yet been investigated nonperturbatively.

\section{Acknowledgments}

We would like to thank Larry Yaffe and Berndt Mueller for useful conversations. This work was supported, in part, by the U.S. Department of Energy under Grant Nos. DEFG02-97ER41027, by the National Sciences and Engineering Research Council of Canada, and by le Fonds Nature et Technologies du Québec.

\section{APPENDIX A: INVERSE BREMSSTRAHLUNG}

In this appendix, we consider the $2 \rightarrow 1$ process of inverse bremsstrahlung, catalyzed by the soft, non-perturbative field, as depicted in Fig. 5. We will treat a case slightly more general than that depicted in the figure, namely the case of one particle with momentum $k \sim \Lambda$ and another particle with a momentum $k^{\prime}$ larger than $m_{\text {soft }}$, up to and including the scale $\Lambda$. We will find that the coherence time of this process is longer than the mean free time between collisions of the particles with the soft background (via Fig. 4), and so we will have to account for the Landau-Pomeranchuk Migdal (LPM) effect [25] in estimating the rate of inverse bremsstrahlung.

For an initial estimate, however, let us momentarily ignore the LPM effect and consider the diagram of Fig. 5 in isolation. Bremsstrahlung in a soft background is a smallangle, nearly-collinear process. Parametrically, the rate for bremsstrahlung or inverse bremsstrahlung may be estimated as (i) the rate for small-angle scattering from the background field (Fig. 4), times (ii) a factor of $g^{2}$ for absorbing or emitting the additional gluon in Fig. 5. times (iii) an initial or final state factor of $f$ or $1+f$ for that gluon, and (iv) a momentum integral $d k^{\prime} / k^{\prime}$ (responsible for the logarithmically large rate of soft bremsstrahlung emission in vacuum). As discussed in Sec. III the rate for small-angle scattering from the non-perturbative background is $O\left(m_{\text {soft }}\right)$. This gives

$$
\Gamma_{(\text {no LPM })} \sim g^{2} m_{\text {soft }} \int \frac{d k^{\prime}}{k^{\prime}} f\left(k^{\prime}\right)
$$

for (inverse) bremsstrahlung. To find the rate for a particle with momentum $k \sim \Lambda$ to substantially change its energy, we must correct for two things. First, absorbing a momentum 
$k^{\prime} \lesssim k$ makes only an order $k^{\prime} / k$ relative change to $\boldsymbol{k}$. Second, it is the difference between the rates of absorption and emission of a particle of momentum $k^{\prime}$ which is relevant. This involves the difference $f(k)-f\left(k+k^{\prime}\right) \sim f(k) k^{\prime} / k$. Therefore we must introduce two powers of $\left(k^{\prime} / k\right)$ into the estimate above, to get

$$
\Gamma_{k \rightarrow 2 k,(\mathrm{no} \mathrm{LPM})} \sim g^{2} m_{\mathrm{soft}} \int \frac{d k^{\prime}}{k^{\prime}}\left(\frac{k^{\prime}}{k}\right)^{2} f\left(k^{\prime}\right) \sim m_{\mathrm{soft}} \int_{m_{\mathrm{soft}}}^{\Lambda}\left(\frac{m_{\mathrm{soft}}}{k^{\prime}}\right)^{\nu} \frac{k^{\prime} d k^{\prime}}{k^{2}},
$$

for doubling energy through inverse bremsstrahlung. For $\nu \leq 2$, this is dominated by $k^{\prime} \sim \Lambda$, and gives $\Gamma \sim m_{\text {soft }}^{1+\nu} \Lambda^{-\nu}$ (up to logarithms when $\nu=2$ ). If we equated the power of $\Lambda$ in (A2) with that in (2.7), we would obtain $\nu=2$, which would imply that this process was parametrically just as important as the multiple soft scattering treated in Sec. III However, the rate (A1) is an overestimate; we must account for the LPM effect.

In Abelian theories, LPM suppression occurs because (i) when a charged particle scatters, the probability that a photon will be bremsstrahlung emitted is not very sensitive to the scattering angle, and (ii) an emitted photon of a given wavelength cannot resolve the difference between a single collision and $N$ collisions if the $N$ collisions are close enough together. ${ }^{10}$ The largest such $N$ can be determined parametrically by equating (i) the time $N \tau$ for $N$ consecutive scatterings of the form of Fig. 4, where $\tau$ is the mean free time between scatterings, with (ii) the formation time $1 /(\delta E)$ for the complete $1 \rightarrow 2$ bremsstrahlung (or $2 \rightarrow 1$ inverse bremsstrahlung) process, where $\delta E$ is the off-shellness of the energy during the process, which is

$$
\delta E=\left|E_{1}+E_{2}-E_{*}\right|
$$

for a $2 \leftrightarrow 1$ process with $\boldsymbol{k}_{1} \boldsymbol{k}_{2} \leftrightarrow \boldsymbol{k}_{*}$. For the reasons of resolution already mentioned, each $N$ collisions are equivalent to a single collision in terms of the probability to induce bremsstrahlung, and so the rate $\Gamma$ for (inverse) bremsstrahlung processes is a factor of $N$ smaller than the naive estimate (A1) which treats every scattering as independent.

The same sort of estimate works for gluon bremsstrahlung in non-Abelian gauge theories. Since bremsstrahlung is an almost collinear process, we can take $k_{\perp} \ll k$, where $\perp$ is relative to the collinear axis, and approximate (A3) for $k \gg m_{\text {soft }}$ as

$$
\frac{m_{1}^{2}+k_{1 \perp}^{2}}{2 k_{1}}+\frac{m_{2}^{2}+k_{2 \perp}^{2}}{2 k_{2}}-\frac{m_{*}^{2}+k_{* \perp}^{2}}{2 k_{*}} .
$$

The main difference between photon bremsstrahlung in QED and gluon bremsstrahlung in QCD is that, in QCD, any of the three particles (including the bremsstrahlung gluon) can undergo the scattering. Therefore we may take each $k_{\perp}^{2} \sim N m_{\text {soft }}^{2}$, in which case $\delta E$ is dominated by the smallest energy particle, which is $k^{\prime}$ in our case. Further, $m^{2} \sim m_{\text {soft }}^{2} \ll k_{\perp}^{2}$ can be dropped, giving

$$
\delta E \sim \frac{k_{\perp}^{2}}{k^{\prime}} \sim \frac{N m_{\text {soft }}^{2}}{k^{\prime}} .
$$

Plugging into (A4), and equating the scattering time $1 / \delta E$ to $N \tau$ as outlined previously, we have

$$
N \tau \sim \frac{1}{\delta E} \sim \frac{k^{\prime}}{N m_{\mathrm{soft}}^{2}}
$$

$\overline{10}$ For a review of the LPM effect in photon bremsstrahlung, see Ref. [26]. For a discussion by the present authors of LPM suppression in non-Abelian plasmas near equilibrium, see Refs. 24, 27. For a partial selection of earlier discussion of the LPM effect in non-Abelian gauge theories, see Refs. 28]. 
Solving for $N$, and using the fact that $\tau \sim 1 / m_{\text {soft }}$ for the individual scattering processes of Fig. 4, determines

$$
N \sim \sqrt{k^{\prime} / m_{\text {soft }}}
$$

Therefore, the rate is reduced by a factor of $\left(m_{\text {soft }} / k^{\prime}\right)^{1 / 2}$, which is a large suppression for $k^{\prime} \gg m_{\text {soft }}$. This modifies Eq. (A2) to,

$$
\Gamma_{k \rightarrow 2 k,(\mathrm{LPM})} \sim m_{\mathrm{soft}} \int_{m_{\mathrm{soft}}}^{\Lambda}\left(\frac{m_{\mathrm{soft}}}{k^{\prime}}\right)^{(\nu+1 / 2)} \frac{k^{\prime} d k^{\prime}}{k^{2}} .
$$

This expression is dominated by small $k^{\prime}$ if $\nu>3 / 2$. The $k^{\prime} \sim \Lambda$ edge of the range of integration provides $\Gamma \sim \Lambda^{-\nu-1 / 2}$, which combined with (2.7) would now yield $\nu=7 / 4$. Therefore, hard bremsstrahlung is not a competitive process. However, the $k^{\prime} \sim m_{\text {soft }}$ edge of the integration range gives $\Gamma \sim m_{\text {soft }}^{3} / \Lambda^{2}$, which combines with Eq. (2.7) to give $\nu=2$. Absorption of an excitation with energy of order $m_{\text {soft }}$ is competitive with (and possibly indistinguishable from) scattering from such excitations, but inverse bremsstrahlung of all harder scales is subdominant.

[1] R. Baier, A. H. Mueller, D. Schiff and D. T. Son, “'Bottom-up' thermalization in heavy ion collisions," Phys. Lett. B 502, 51 (2001) hep-ph/0009237.

[2] P. Arnold, J. Lenaghan and G. D. Moore, "QCD plasma instabilities and bottom-up thermalization," JHEP 08 (2003) 002 hep-ph/0307325.

[3] A. H. Mueller, A. I. Shoshi and S. M. H. Wong, "A possible modified 'bottom-up' thermalization in heavy ion collisions," hep-ph/0505164.

[4] D. Bodeker, "The impact of QCD plasma instabilities on bottom-up thermalization," hep-ph/0508223

[5] E. S. Weibel, "Spontaneously growing transverse waves in a plasma due to an anisotropic velocity distribution," Phys. Rev. Lett. 2, 83 (1959).

[6] U. W. Heinz, "Quark-gluon transport theory," Nucl. Phys. A 418, 603C (1984).

[7] Y. E. Pokrovsky and A. V. Selikhov, "Filamentation in a quark-gluon plasma," JETP Lett. 47, 12 (1988) [Pisma Zh. Eksp. Teor. Fiz. 47, 11 (1988)]; "Filamentation in quark plasma at finite temperatures," Sov. J. Nucl. Phys. 52, 146 (1990) [Yad. Fiz. 52, 229 (1990)]; "Filamentation in the quark-gluon plasma at finite temperatures," Sov. J. Nucl. Phys. 52, 385 (1990) [Yad. Fiz. 52, 605 (1990)].

[8] O. P. Pavlenko, "Filamentation instability of hot quark-gluon plasma with hard jet," Sov. J. Nucl. Phys. 55, 1243 (1992) [Yad. Fiz. 55, 2239 (1992)].

[9] S. Mrówczyński, "Stream instabilities of the quark-gluon plasma," Phys. Lett. B 214, 587 (1988); "Plasma instability at the initial stage of ultrarelativistic heavy ion collisions," 314, 118 (1993); "Color collective effects at the early stage of ultrarelativistic heavy ion collisions," Phys. Rev. C 49, 2191 (1994); "Color filamentation in ultrarelativistic heavy-ion collisions," Phys. Lett. B 393, 26 (1997) hep-ph/9606442; J. Randrup and S. Mrówczyński, "Chromodynamic Weibel instabilities in relativistic nuclear collisions," Phys. Rev. C 68, 034909 (2003) nucl-th/0303021.

[10] S. Mrówczyński and M. H. Thoma, "Hard loop approach to anisotropic systems," Phys. Rev. D 62, 036011 (2000) hep-ph/0001164; 
[11] P. Romatschke and M. Strickland, "Collective modes of an anisotropic quark gluon plasma," Phys. Rev. D 68, 036004 (2003) hep-ph/0304092.

[12] P. Arnold and J. Lenaghan, "The Abelianization of QCD plasma instabilities," Phys. Rev. D 70, 114007 (2004) hep-ph/0408052.

[13] P. Arnold, J. Lenaghan, G. D. Moore and L. G. Yaffe, "Apparent thermalization due to plasma instabilities in quark gluon plasma," Phys. Rev. Lett. 94, 072302 (2005) nucl-th/0409068.

[14] A. Rebhan, P. Romatschke and M. Strickland, "Hard-loop dynamics of non-Abelian plasma instabilities," Phys. Rev. Lett. 94, 102303 (2005) hep-ph/0412016.

[15] A. Dumitru and Y. Nara, "QCD plasma instabilities and isotropization," hep-ph/0503121.

[16] P. Arnold, G. D. Moore and L. G. Yaffe, "The fate of non-abelian plasma instabilities in 3+1 dimensions," Phys. Rev. D 72, 054003 (2005) hep-ph/0505212.

[17] A. Rebhan, P. Romatschke and M. Strickland, "Dynamics of quark-gluon plasma instabilities in discretized hard-loop approximation," hep-ph/0505261.

[18] P. Arnold and G. D. Moore, "QCD plasma instabilities: the non-abelian cascade," hep-ph/0509206.

[19] D. T. Son, "Reheating and thermalization in a simple scalar model," Phys. Rev. D 54, 3745 (1996) hep-ph/9604340.

[20] R. Micha and I. I. Tkachev, "Relativistic turbulence: A long way from preheating to equilibrium," Phys. Rev. Lett. 90, 121301 (2003) hep-ph/0210202.

[21] R. Micha and I. I. Tkachev, "Turbulent thermalization," Phys. Rev. D 70, 043538 (2004) hep-ph/0403101.

[22] A. H. Mueller and D. T. Son, "On the equivalence between the Boltzmann equation and classical field theory at large occupation numbers," Phys. Lett. B 582, 279 (2004) hep-ph/0212198.

[23] V. N. Tsytovich, Lectures on Non-linear Plasma Kinetics (Springer-Verlag, 1995).

[24] P. Arnold, G. D. Moore and L. G. Yaffe, "Effective kinetic theory for high temperature gauge theories," hep-ph/0209353.

[25] A. B. Migdal, "Bremsstrahlung And Pair Production In Condensed Media At High-Energies," Phys. Rev. 103, 1811 (1956); Doklady Akad. Nauk S. S. S. R. 105, 77 (1955); L. D. Landau and I. Pomeranchuk, Dokl. Akad. Nauk Ser. Fiz. 92 (1953) 535; "Electron Cascade Process At Very High-Energies," Dokl. Akad. Nauk Ser. Fiz. 92 (1953) 735; [The last two papers are also available in English in L. Landau, The Collected Papers of L.D. Landau (Pergamon Press, New York, 1965).]

[26] S. Klein, "Suppression of bremsstrahlung and pair production due to environmental factors," Rev. Mod. Phys. 71, 1501 (1999) hep-ph/9802442.

[27] P. Arnold, G. D. Moore and L. G. Yaffe, "Photon and gluon emission in relativistic plasmas," JHEP 0206, 030 (2002) hep-ph/0204343.

[28] B. G. Zakharov, "Fully quantum treatment of the Landau-Pomeranchuk-Migdal effect in QED and QCD," JETP Lett. 63, 952 (1996) hep-ph/9607440; "Light-cone path integral approach to the Landau-Pomeranchuk-Migdal effect," Phys. Atom. Nucl. 61 (1998) 838 [Yad. Fiz. 61 (1998) 924] hep-ph/9807540; R. Baier, Y. L. Dokshitzer, S. Peigne and D. Schiff, "Induced gluon radiation in a QCD medium," Phys. Lett. B 345, 277 (1995) hep-ph/9411409; R. Baier, Y. L. Dokshitzer, A. H. Mueller, S. Peigne and D. Schiff, "Radiative energy loss of high energy quarks and gluons in a finite-volume quark-gluon plasma," Nucl. Phys. B 483, 291 (1997) hep-ph/9607355; R. Baier, Y. L. Dokshitzer, A. H. Mueller, S. Peigne and D. Schiff, "Radiative energy loss and p(T)-broadening of high energy partons in nuclei," 484, 265 (1997) hep-ph/9608322 ; R. Baier, D. Schiff and B. G. Zakharov, "Energy loss in perturbative QCD," 
Ann. Rev. Nucl. Part. Sci. 50, 37 (2000) hep-ph/0002198. 